\title{
Clinicopathologically problematic melanocytic tumors: a case-based review
}

\author{
Zoe Apalla ${ }^{1}$, Christina Nikolaidou ${ }^{1,2}$, Aimilios Lallas ${ }^{1}$, Elena Sotiriou ${ }^{1}$, Elizabeth Lazaridou ${ }^{3}$, \\ Ioannis Venizelos ${ }^{2}$, Mattheos Bobos ${ }^{4}$, Efstratios Vakirlis ${ }^{1}$, Demetrios Ioannides ${ }^{1}$, Gerardo Ferrara ${ }^{5}$
}

1 First Dermatology Department, Aristotle University of Thessaloniki, Greece

2 Anatomic Pathology Unit, Hippokration Hospital, Thessaloniki, Greece

3 Second Dermatology Department, Aristotle University of Thessaloniki, Greece

4 Microdiagnostics Pathology Laboratory, Thessaloniki, Greece

5 Anatomic Pathology Unit, Hospital of Macerata, Italy

Key words: melanoma, dermoscopy, skin cancer, nevus, spitzoid

Citation: Apalla Z, Nikolaidou C, Lallas A, Sotiriou E, Lazaridou E, Venizelos I, Bobos M, Vakirlis E, Ioannides D, Ferrara G.

Clinicopathologically problematic melanocytic tumors: a case-based review. Dermatol Pract Concept. 2018;8(4):306-313. DOI: https://doi. org/10.5826/dpc.0804a12

Received: February 28, 2018; Accepted: May 31, 2018; Published: October 31, 2018

Copyright: (02018 Apalla et al. This is an open-access article distributed under the terms of the Creative Commons Attribution License, which permits unrestricted use, distribution, and reproduction in any medium, provided the original author and source are credited.

Funding: None.

Competing interests: The authors have no conflicts of interest to disclose.

All authors have contributed significantly to this publication.

Corresponding author: Zoe Apalla, MD, First Dermatology Department, Aristotle University of Thesssaloniki, 124 Delfon St, Thessaloniki, Greece. Email: zoimd@yahoo.gr

ABSTRACT Background: In spite of recent advances in the histopathological and molecular diagnosis of melanocytic neoplasms, a certain proportion of these lesions remain a daunting challenge for both the clinician and the pathologist.

Objectives: To emphasize the importance of close collaboration between clinicians and pathologists in case of problematic melanocytic lesions.

Patients: We report and discuss 5 problematic scenarios of melanocytic lesions, including tumoral melanosis, nevoid melanoma, lentiginous melanoma, spitzoid melanoma and BAPoma that may pose diagnostic difficulties in our practice.

Conclusions: Clinico-dermoscopic-pathological correlation, with incorporation of all the available data, in problematic melanocytic skin neoplasms is of paramount importance for accurate diagnosis.

\section{Introduction}

Early detection of melanoma is of paramount importance for the patient. In recent decades, relevant scientific advances in the field of pathogenesis, epidemiology, and evolution of melanocytic lesions, in conjunction with the development of new diagnostic techniques, have enhanced our ability to diagnose melanoma early and accurately $[1,2]$. However, the final diagnosis still relies on the correct histopathological assessment, which is considered the cornerstone in melanoma diagnosis [3].

In reality, even though the majority of the histological diagnoses of melanocytic lesions can be established with a high degree of confidence, there is still a certain subset of 


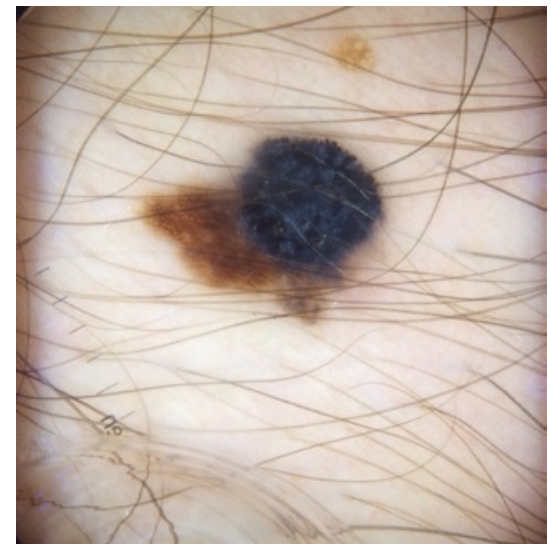

Figure 1. Case 1. Dermoscopy shows an asymmetric lesion exhibiting a brown atypical network, a black-blue blotch with peripheral streaks, and white veil with foci of "peppering." [Copyright: (C2018 Apalla et al.]

melanocytic neoplasms that raise difficulties for both pathologists and clinicians [3].

In the current case-based review, we discuss problematic scenarios that emanate from clinico-dermoscopic-pathological discrepancies. Overcoming the obstacles and establishing a diagnostic approach that facilitates case management is of paramount importance. The success of this approach relies on close collaboration between the clinician and the pathologist, and it presupposes incorporation and exploitation of all available data.

\section{Case 1}

A 53-year-old man attended our outpatient department seeking medical advice for a gradually enlarging pigmented lesion, located on his upper chest. There was no personal or family history of melanoma and the patient did not suffer from dysplastic nevus syndrome or from a high number of moles. Clinical examination revealed an asymmetric plaque measuring $1.2 \mathrm{~cm}$ at its largest diameter. On dermoscopy (Figure 1) the lesion was highly suspicious: it morphologically consisted of 2 different components, 1 characterized by the presence of a brown atypical network, signifying a melanocytic lesion occupying the dermoepidermal junction, and 1 dis-

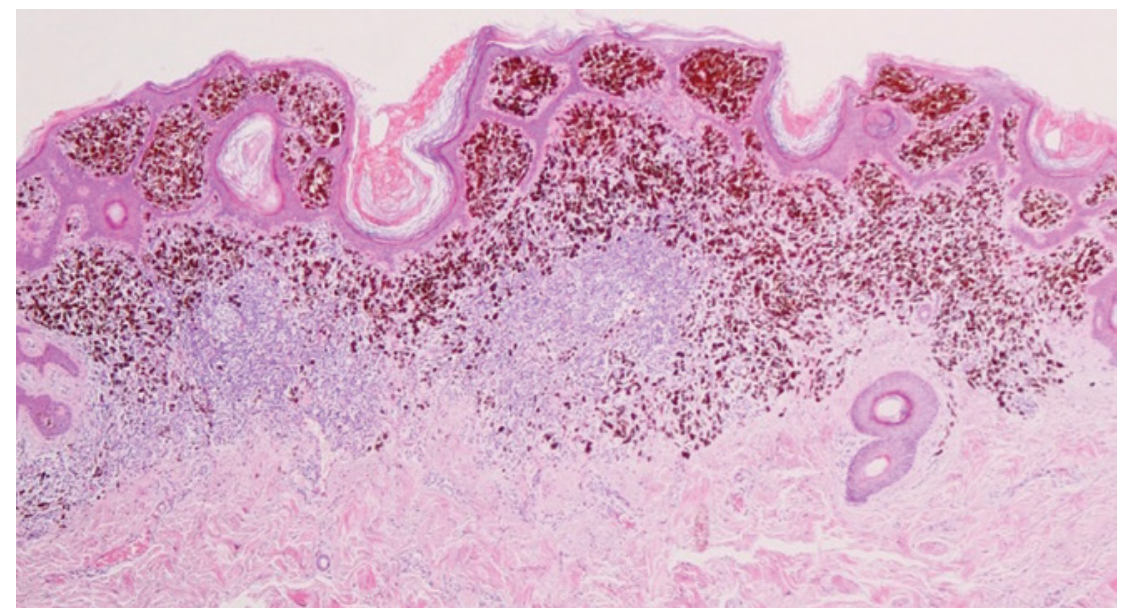

Figure 2. Case 1 . H\&E, original magnification $\times 20$. Extensive regression typified by nodular aggregates of dermal melanophages, focal fibrosis, dense infiltrate of lymphocytes, and absence of melanocytes, corresponding to the black-blue blotch seen in dermoscopy. [Copyright: (C2018 Apalla et al.]

playing almost all of the dermoscopic criteria of melanoma. In particular, there was a black-blue blotch with peripheral streaks, white veil, and foci of "peppering." The lesion was immediately excised under the clinical diagnosis of melanoma and was histologically examined. Surprisingly, the histological report was not conclusive of a melanoma.

Analytically, the pathologist reported the presence of a melanocytic lesion displaying characteristics of a "dysplastic nevus" with a wide area of regression (nodular aggregates of dermal melanophages, focal fibrosis, dense infiltrate of lymphocytes, and absence of melanocytes). Although the residual lesion did not disclose clear-cut features of malignancy, the clinical features, along with the presence of nodular aggregates of melanophages (tumoral melanosis), pointed to a completely regressed melanoma (Figure 2).

\section{Case 2}

A 42-year-old man attended the skin cancer unit seeking medical advice for a verrucous hyperkeratotic lesion of 6 months' duration, located on the abdomen. Clinical examination revealed a brittle, hard in palpation, $0.8-\mathrm{cm}$ brownish papule. On dermoscopy, we observed a central keratin plug, covering a papillomatous lesion. Within the keratin, there were hemorrhagic crusts. Atypical linear vessels transcended the papillomatous projections. The vessels mimicked at one site the hairpin vessels seen in keratoacanthoma, common warts, and irritated seborrheic keratosis (Figure 3). In addition, there were focal strands of pigmentation projecting in a radial manner from the center of the lesion. The presence of atypical vessels and the inability to establish a specific diagnosis forced us to surgically excise the lesion. In our differential diagnosis we mainly included inflamed seborrheic keratosis, common wart, and keratoacanthoma.

On hematoxylin and eosin (H\&E) stained slides, at low power the lesion resembled a papillomatous benign intradermal nevus. It was characterized by epidermal hyperplasia, elongation of rete ridges, and overlying hyperkeratosis. However, higher magnifications revealed that the lesion was hypercellular, with intraepidermal and dermal proliferation of atypical melanocytes (Figure 4a). Although resembling nevus cells, dermal melanocytes displayed hyperchromatic mildly pleomorphic nuclei, scanty cytoplasm, and occasionally prominent nucleoli. Sparse mitoses in the dermis were occasionally recognized (Figure 4b). Dermal melanocytes revealed an increased proliferative 


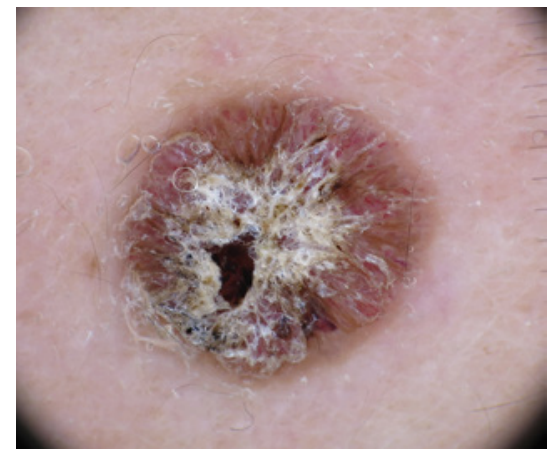

Figure 3. Case 2. Dermoscopy shows a central keratin plug covering a papillomatous lesion. Papillomatous projections are transcended by atypical linear vessels, while there are also strands of pigmentation distributed in a radial manner from the center of the lesion. [Copyright: @2018 Apalla et al.]

index (Ki-67) of $12 \%$. These findings were consistent with a verrucous nevoid melanoma.

\section{Case 3}

A 73-year-old woman visited us for a newly developed, gradually enlarging, "freckle" on her shin, asking treatment for cosmetic reasons. The lesion was an asymmetric, $2.5-\mathrm{cm}$, well-defined patch, consisting of a light-brown upper part and a darker lower part (Figure 5a). Based on naked-eye clinical examination we thought of solar lentigo. However, dermoscopy was inconclusive, characterized by a multicomponent pattern with sites of faint pigment network, brownish structureless areas, "fingerprinting," as well as focal distribution of atypical clods (Figure 5b). Considering the ambiguous features, a melanocytic proliferation could not be excluded. In this context, we decided to perform a dermoscopy-guided incision biopsy.

The incision biopsy aimed to differentiate between a melanocytic and a nonmelanocytic lesion; thus, even if histopathology was in favor of a junctional nevus, the lesion was finally excised. It is important to specify that the pathologist who examined the slides of the initial incision biopsy was unaware of the clinical and dermoscopic picture. Histopathological examination of the

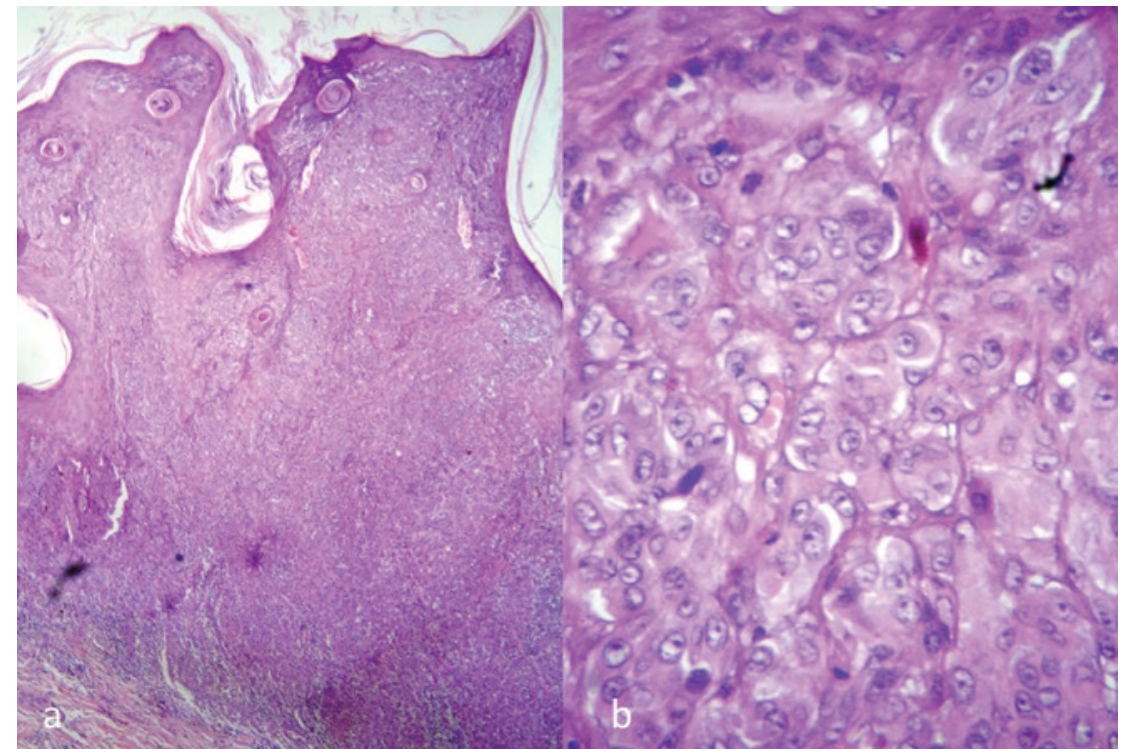

Figure 4. Case 2. (a) On histology (H\&E, original magnification $\times 10)$, verrucous nevoid melanoma displays epidermal hyperplasia, elongation of rete ridges, and overlying hyperkeratosis resembling dermal nevus. (b) However, higher magnifications $(\times 20)$ reveal a hypercellular lesion with intraepidermal and dermal proliferation of atypical nonmaturing melanocytes. [Copyright: @2018 Apalla et al.]

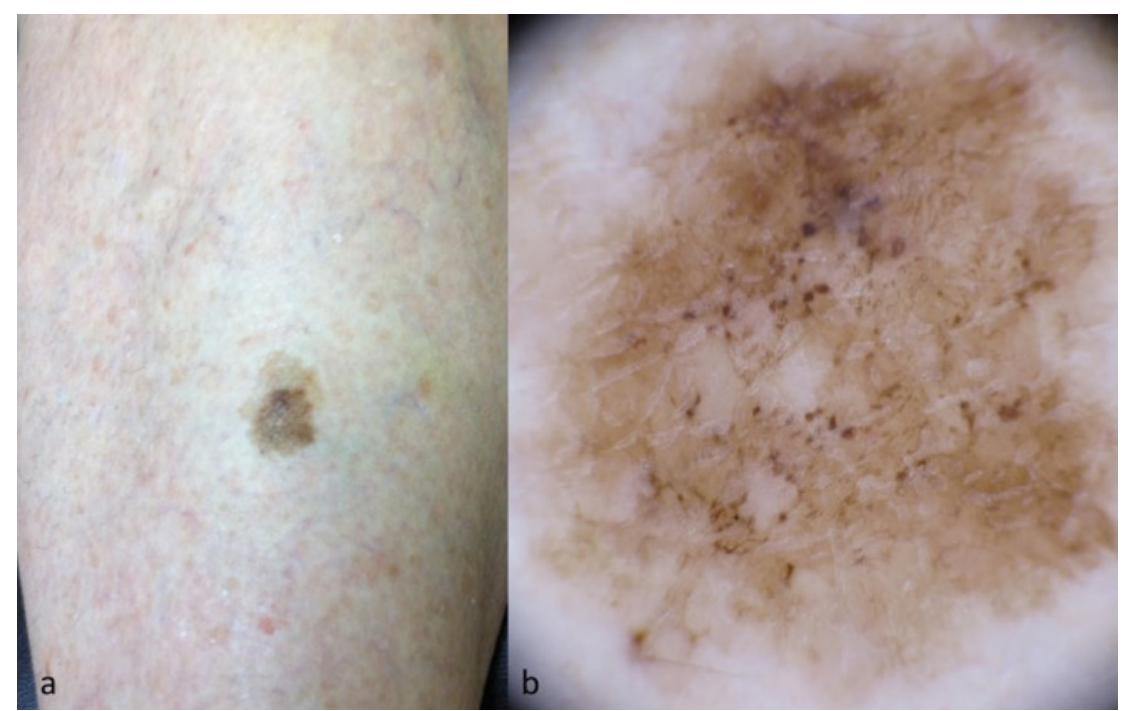

Figure 5. Case 3. (a) Clinically there is an asymmetric, well-defined patch, consisting of a light-brown upper part and a darker lower part. (b) Dermoscopy shows a multicomponent pattern, with sites of faint pigment network, brownish structureless areas, "fingerprinting," as well as focal distribution of atypical clods. [Copyright: (O2018 Apalla et al.]

excision specimen revealed a large melanocytic tumor with a relatively regular epidermal hyperplasia and many single melanocytes tightly packed at the junction (Figure 6). Also considering the age of the patient, the evolution of the lesion, and the dermoscopic characteristics, the lesion was finally diagnosed as a lentiginous melanoma on sun-damaged skin of the elderly.

\section{Case 4}

During the annual skin check of a 19-year-old female albino patient we noticed a newly developed red lesion on the trunk. Clinically it was a $0.4-\mathrm{cm}$ hemispheric papule of elastic constitution (Figure 7a). Dermoscopically it was characterized by the presence of in-focus linear arborizing vessels in a pinkish background (Figure $7 \mathrm{~b}$ ). In view 
of the dermoscopic findings and taking into consideration that the patient suffered from oculocutaneous albinism, our first clinical diagnosis was a nodular basal cell carcinoma, while we could not exclude an amelanotic melanoma. Based on the latter, we decided to surgically excise it.

On histology, the lesion demonstrated symmetric polypoid architecture with a minimal junctional component. The dermal component was composed of a population of large epithelioid cells with abundant amphophilic cytoplasm, large nucleoli, and some degree of pleomorphism. In addition, there was a "brisk" lymphocytic infiltrate (Figure 8). The differential diagnosis comprised a "halo" Spitz nevus; however, the lesion was not haloed clinically and no true "spitzoid" feature was evident dermoscopically. A re-evaluation of all these features raised the hypothesis that the lesion might be a classic Wiesner nevus (BAPoma). The latter diagnosis was confirmed by the loss of nuclear BAP-1 expression in the epithelioid cells with immunohistochemistry. The patient did not exhibit multiple fibroma-like nevi and her family history was not suggestive of a familial cancer syndrome. Germline genetic testing was negative.

\section{Case 5}

A 38-year-old man attended the Skin Cancer Unit requesting a "mole check." During the clinical examination we observed a dark $0.5 \mathrm{~cm}$ macule on his right wrist. The patient mentioned that this was a rapidly enlarging, recent lesion. On dermoscopy, we recognized an asymmetric, heavily pigmented darkbrown lesion with radial streaks at the periphery (Figure 9).

At the lower part of the lesion there was an area of gray color, in keeping with early regression. The overall morphological pattern was suggestive of a spitzoid melanocytic neoplasm. The lesion was surgically excised.

On low magnification the tumor appeared as heavily pigmented, com-

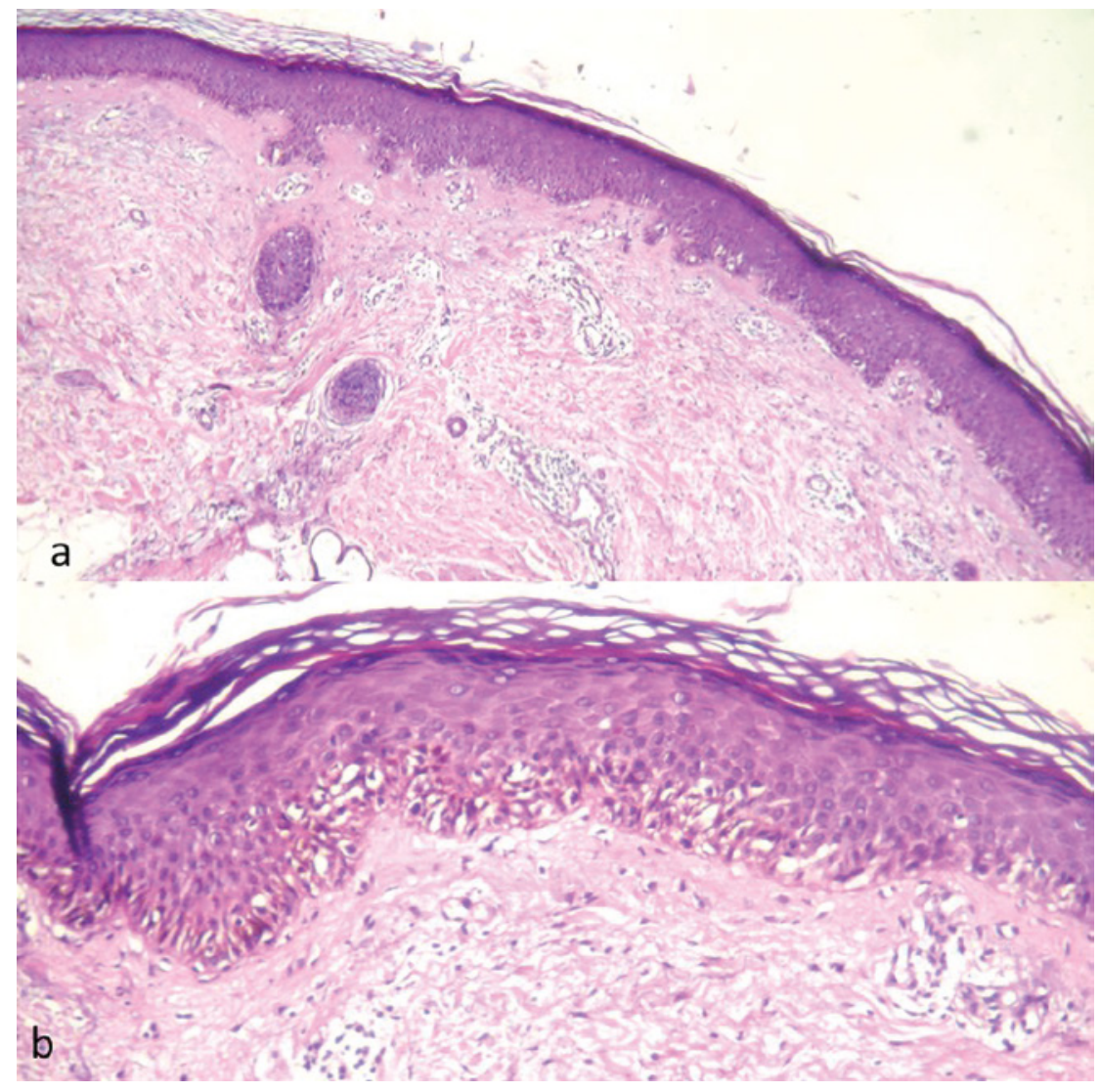

Figure 6. Case 3. Histopathological examination reveals a large melanocytic tumor (H\&E, original magnification $\times 10$ ) with a relatively regular epidermal hyperplasia and many single melanocytes tightly packed at the junction (×20). [Copyright: (C2018 Apalla et al.]

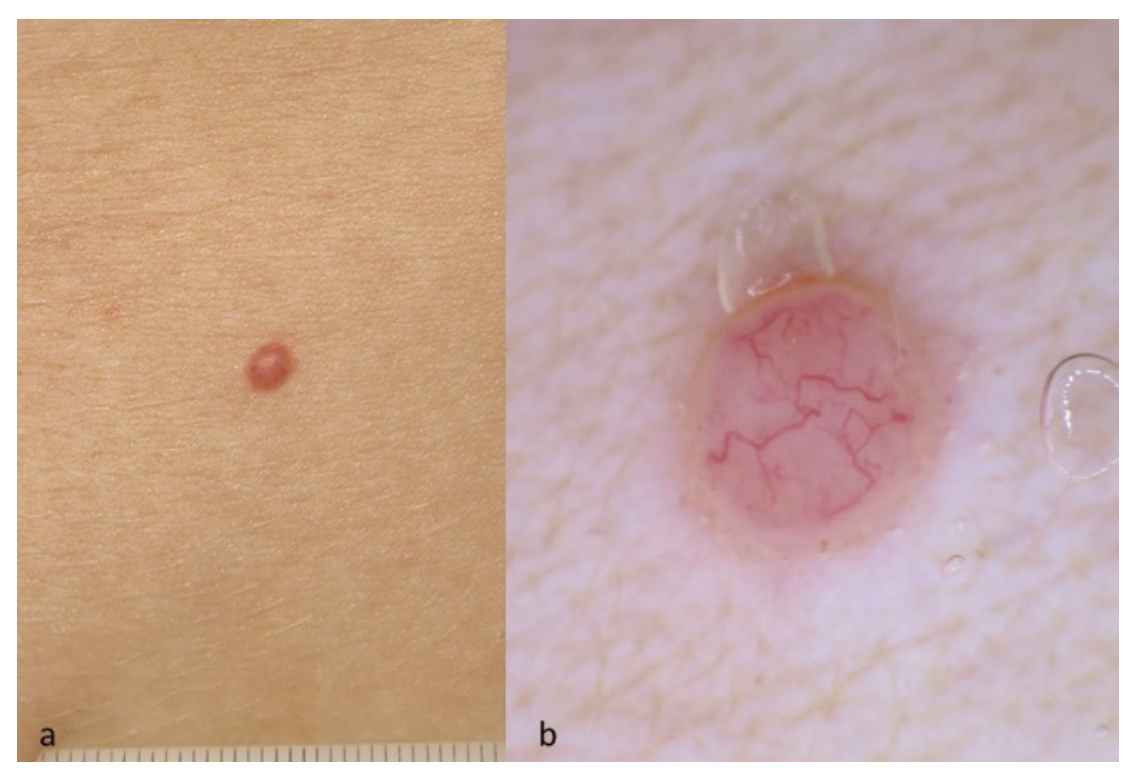

Figure 7. Case 4. (a) Clinically there is a hemispheric pink papule. (b) Dermoscopy shows in-focus linear arborizing vessels in a pinkish background. [Copyright: (C2018 Apalla et al.]

pound, with asymmetric architecture and focal pagetoid configuration (Figure 10a). It consisted of predominantly spindled melanocytes arranged in discohesive nests, with a few mitotic figures
(Figure 10b-10d). These features, taken together with the clinical and the dermoscopic picture, favored the diagnosis of atypical Spitz nevus over spitzoid melanoma. 


\section{Discussion}

In the current article, we use 5 real-life clinical examples to highlight difficulties in clinicopathological correlation of certain groups of melanocytic tumors, namely, the fully regressed melanoma, the verrucous nevoid melanoma, the spitzoid tumor, the lentiginous melanoma of sun-exposed areas in the elderly, as well as the quite recently introduced melanocytic entity of BAPoma. All the aforementioned lesions may raise diagnostic difficulties for the dermatologist and/or the pathologist, with subsequent implications in case management [4].

Spontaneous regression is a relatively common event in melanoma, characterized by an overall incidence that ranges from $10 \%$ to $35 \%$. The biological mechanism behind regression has not been completely elucidated; however, it seems that it is mainly attributed to host-immune-mediated responses that ultimately result in partial or complete disappearance of the neoplasm [5,6].

From the clinical point of view, regression may be seen as areas of depigmentation, and less often as areas of hyperpigmentation. In the former case the most evident pattern in dermoscopy includes whitish or pinkish areas, as well as gray dots on a whitish background, the so-called "peppering." In the case of hyperpigmentation-mostly present in the early regression phase-

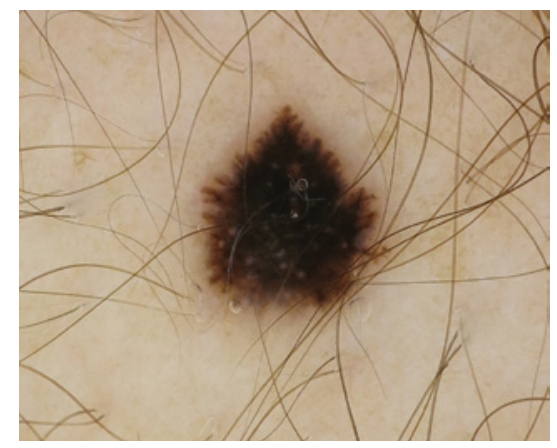

Figure 9. Case 5. An asymmetric, heavily pigmented dark-brown lesion with radial streaks at the periphery. A tiny area of gray color is in keeping with early regression. [Copyright: @2018 Apalla et al.]
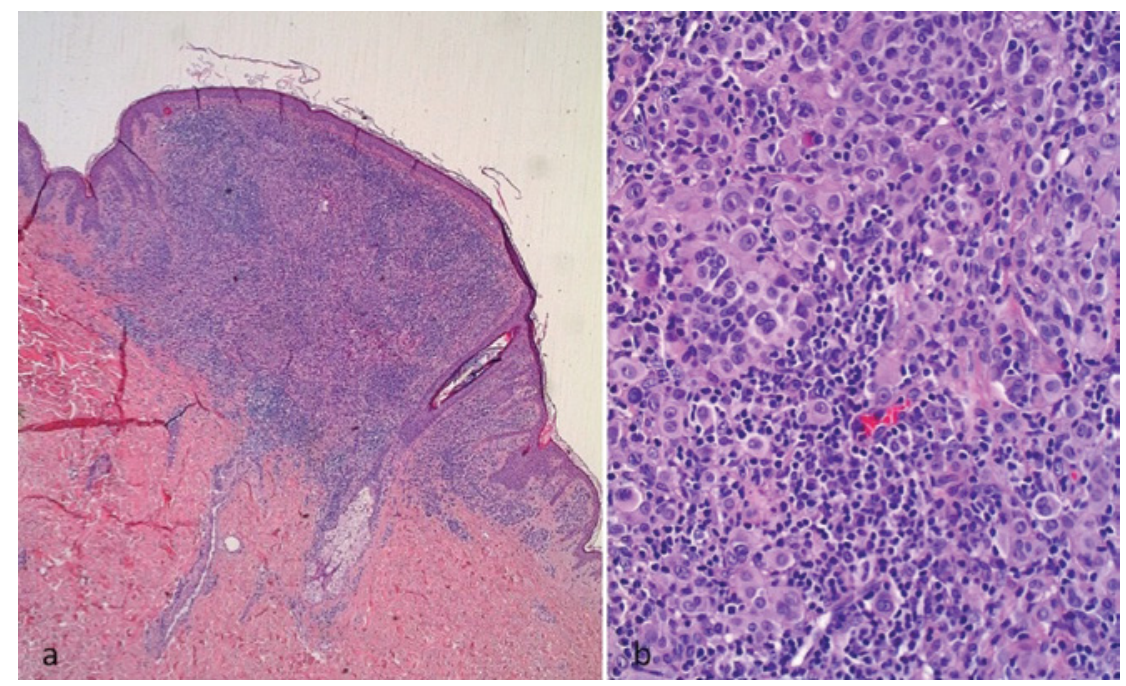

Figure 8. Case 4. On histology (H\&E, original magnification $\times 10)$, the lesion demonstrates symmetric polypoid architecture with a minimal junctional component. Large epithelioid cells with abundant amphophilic cytoplasm, large nucleoli, and some degree of pleomorphism (×20). [Copyright: (C2018 Apalla et al.]

the predominant dermoscopic color is blue, gray, and/or black [7,8].

From the clinician's perspective, the most diagnostically challenging scenario is the late-phase, fully regressed melanoma, appearing with depigmentation

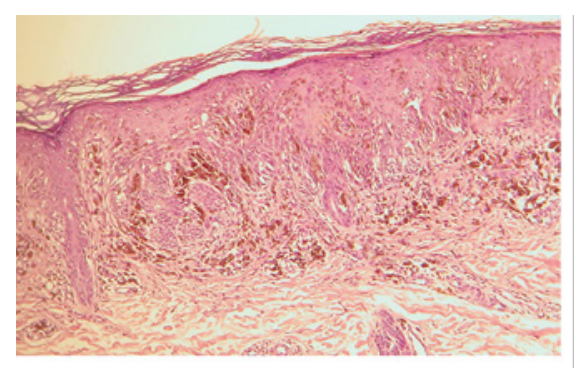

(a)
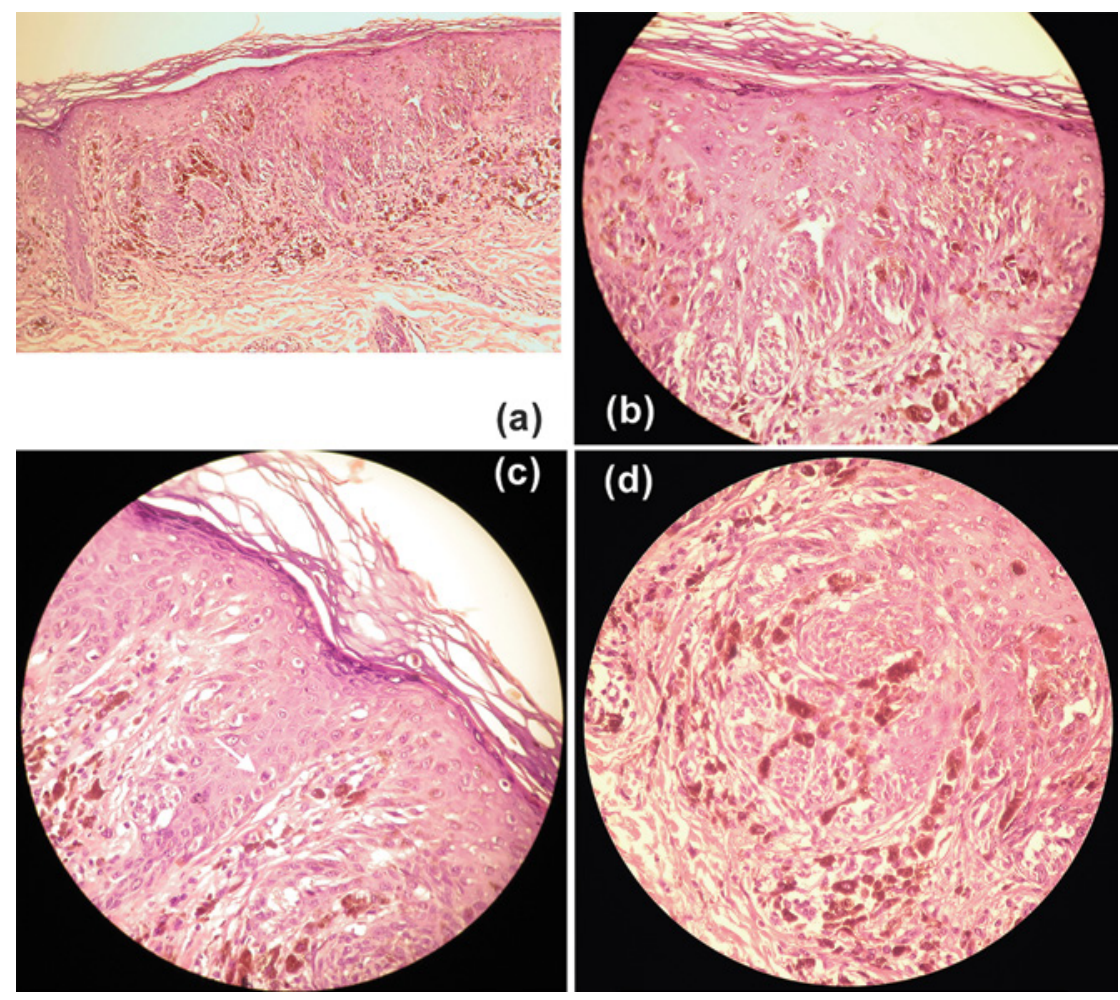

Figure 10. Case 5. The tumor appears as heavily pigmented, compound, with asymmetric architecture and focal pagetoid configuration. It consisted of predominantly spindled melanocytes arranged in discohesive nests, with a few mitotic figures (H\&E, original magnification $\times 20)$. [Copyright: $@ 2018$ Apalla et al.] and peppering, since this dermoscopic pattern is not considered pathognomonic for melanoma and it can be easily misinterpreted as a lichen planus-like keratosis or as a regressed nevus [7-9]. On the other hand, a heavily pigmented 
area in dermoscopy, as in our patient, certainly raises the level of suspicion, making the clinical diagnosis of melanoma the most probable.

Despite the lack of a standard definition for histological regression, it is generally typified by a variable decrease in the number of melanocytes and the concomitant presence of an immune host response, comprising dermal fibrosis, inflammatory cell infiltrate, melanophages, vascular ectasia, epidermal attenuation, with or without apoptosis of keratinocytes, or melanocytes. There are significant inconsistencies concerning the histological assessment of regression, with no universally accepted set of criteria. The American Joint Committee on Cancer classification system recommends $75 \%$ as the cutoff for defining focal vs extensive regression. The latter was based on studies reporting a higher rate of metastasis among melanomas with $\geq 75 \%$ regression $[5,6]$.

Apparently, for (dermato-) pathologists, the most problematic context is the rare case of a completely regressed primary cutaneous melanoma. On certain occasions it is exceedingly difficult to establish a definite melanoma diagnosis, especially in the absence of a reliable clinical history of a rapidly changing pigmented lesion [10].

In the second case we describe a nevoid verrucous melanoma clinically and dermoscopically mimicking a benign keratinizing tumor. A seborrheic keratosis resembling a melanoma is not uncommon, while the opposite-a melanoma mimicking a seborrheic keratosis-is considered rare $[11,12]$. This uncommon latter scenario hides serious risks for the clinician and the patient. The lack of clear-cut melanoma criteria and the presence of "benign" features may falsely lead to the diagnosis of a seborrheic keratosis or common wart. In this context, the biopsy and the histological assessment may be skipped, resulting in a significant delay in the diagnosis of melanoma, with unpredictable consequences to the patient's health. In addition, inappropriate treatment based on the mistaken diagnosis may complicate further the already noisome scenario [11-14].

It has been shown in the literature that seborrheic keratosis-like melanomas are clinically and dermoscopically challenging. However, dermoscopy has proved to be particularly useful, since despite the presence of additional seborrheic keratosis dermoscopic features, the identification of pseudopods and/or streaks, the blue-black sign, pigment network, and/or blue-white veil facilitates the correct diagnosis of the majority of the tricky melanomas [14].

The lesion in case 2 is considerably educative also from the histopathological point of view. It turned out to be a verrucous nevoid melanoma, which is a rare variant of melanoma, characterized by deceptive morphology, reminiscent of a benign melanocytic nevus. At scanning magnification these lesions show a strong resemblance to banal dermal nevi. They are well circumscribed, usually with an inconspicuous junctional component and composed of a monomorphous cell population. The deeper margin frequently displays an infiltrating growth pattern [15-18].

Zembowicz et al morphologically analyzed 20 nevoid melanomas and were able to confirm 2 major subtypes, referred to as verrucous and nodular variants [17]. The former variant was characterized by strikingly uniform histological findings. The prototype, identically to our case, was a quite symmetric and well-defined exophytic lesion with hyperkeratosis and papillomatosis, denoting a verrucous architecture. According to the authors, "the most reliable feature distinguishing these lesions from benign nevi is the invariable presence of multiple dermal mitoses, including those within the deeper aspects of the lesions" [17].

Taking into account that nevoid melanoma has the same prognosis as the classic one, the earliest possible diagnosis is exceedingly important. Exhaustive evaluation of any "nevus" displaying unusual morphology minimizes the possibility of misdiagnosis. Significant architectural characteristics of nevoid melanoma are the sheet-like growth pattern and/or the presence of expansile nodules. In terms of cytology, the main diagnostic features include lack of or only minimal maturation, mild nuclear pleomorphism with focal hyperchromasia, and intradermal mitoses, often in a high number [15-18].

The third case in our series deals with a melanoma arising in the background of chronically sun-damaged skin on the lower leg of an elderly woman. Clinically, melanomas involving nonfacial sun-exposed areas with photodamage can raise diagnostic difficulties because they often masquerade as solar lentigines, seborrheic keratosis, or nevi and may be camouflaged among the plethora of surrounding pigmented benign lesions [19].

An additional "trap" when we deal with pigmented lesions in the elderly is associated with the limitations of incisional biopsies. The latter diagnostic approach carries a risk of underdiagnosis, as happened in our case [20]. We decided to perform an incisional biopsy because the lesion was large and located over a body site, at which primary surgical closure could be difficult. However, histopathological assessment of a small part of the lesion was in favor of dysplastic nevus, a diagnosis that was inconsistent with the patient's age and evolution of the lesion. After collaboration of the clinician and the pathologist, as well as implementation of a clinicopathological approach, it was recommended excisional biopsy that was diagnostic of a lentiginous melanoma.

In a study evaluating the clinical and dermoscopic characteristics of melanomas on nonfacial chronically sun-damaged skin, it was shown that $76 \%$ of melanomas were in situ, with lentigo maligna ( $40.9 \%$ ) being the most common histopathological type, followed by superficial spreading melanoma $(22.6 \%)$ and lentiginous melanoma $(10.2 \%)$. With regard to the dermoscopic structures, the most frequent were granu- 
larity and angulated lines. Aggregated dots were observed in $36.6 \%$ of the cases. Based on these observations, the authors concluded that recognition of these patterns to the specific anatomy of nonfacial sun-damaged skin might indicate a melanoma. In our patient, angulated lines, structureless areas of pigmentation, and aggregated dots/globules raised our suspicion for melanoma and prompted us to perform a biopsy and ultimately diagnose the melanoma [19].

Lentiginous melanoma of the elderly is believed by many investigators to represent a distinct subset of melanomas. In general, atypical lentiginous proliferations of melanocytes in elderly individuals are diagnostically challenging: they are occasionally classified as dysplastic nevi, atypical junctional nevi, early melanoma in situ, and premalignant melanosis [20]. King et al reported a case series of lentiginous melanoma, in which the histological features of the initial biopsies resembled dysplastic nevus, or lentiginous nevus [21]. These lesions were characterized by lentiginous melanocytic proliferation along the dermoepidermal junction. The melanocytes were distributed both as single cells, as well as in small nests and displayed cytological atypia [21]. Subsequent re-excisions, as in our case, elucidated the diagnosis of melanoma, given the striking predominance of single junctional melanoctyes over nests. Concerning the management, since there is a risk of progression to invasive melanoma, all lesions displaying features of lentiginous melanoma should be treated accordingly [22].

BAPoma was originally described by Wiesner et al in 2011 [23]. The authors described 2 families with a new syndrome inherited in an autosomal dominant manner, clinically showing multiple pinkish, dome-shaped melanocytic tumors. As opposed to common acquired nevi, melanocytic lesions in the affected individuals histopathologically ranged from nevi with epithelioid melanocytes to atypical melanocytic proliferations with features overlapping with those seen in melanoma. Another observation was that some of the affected family members developed uveal or cutaneous melanoma. Genetic analysis revealed that this phenotype was associated with inactivating germline mutations of the BAP1 gene. The majority of these melanocytic tumors lost the remaining wild-type allele of $B A P 1$ as a result of various somatic alterations. Furthermore, the investigators reported $B A P 1$ mutations in a group of sporadic melanocytic lesions, showing clinical and histological features similar to the familial tumors. Based on these findings, it was suggested that biallelic inactivation of BAP1 is related to a clinically and morphologically distinct type of melanocytic neoplasm [23]. The latter was further reported in subsequent publications, describing sporadic cases of BAPoma, displaying identical clinical and histopathological characteristics [24-27].

Our experience suggests that Wiesner nevus, with the ambiguous clinical and histopathological characteristics, may raise some diagnostic difficulties. Clinically, these tumors develop as slowly growing pink nodules, not suggestive of a specific diagnosis. On the other hand, the dermoscopic pattern of this entity is scantily described in the literature and seems to differ significantly from a banal dermal nevus [24]. For example, in our patient, the in-focus arborizing vessels in dermoscopy strongly suggested a basal cell carcinoma. In the field of histopathology, depending on the grade of atypia present in a Wiesner nevus, the main differentials include Spitz nevus/spitzoid tumors and melanoma [23-27].

The biological classification of melanocytic lesions with BAP1 loss remains controversial. Njauw et al characterized melanocytic neoplasms with germline BAP1 mutations as "severely atypical, reminiscent of nevoid melanoma," and in many cases as "short of frank malignancy, though these lesions clearly lie within the spectrum of nevoid melanomas" [27]. However, these lesions had clinical and histopathological characteristics similar to those presented by Wiesner et al [23] as benign tumors. Better understanding of their biology will enhance our ability to distinguish them from other melanocytic neoplasms and better classify them in the future [24].

The last case is devoted to the highly complex group of spitzoid tumors. It was about 1 century ago when the first report of a peculiar, rapidly growing melanocytic lesion was reported in the literature [28]. However, it seems that there is still a long way to go until the ongoing enigma is resolved, which practically refers to our inability to predict the biological behavior of these lesions based on their clinical, dermoscopic, histopathological, and morphological characteristics. The numerous and sometimes diverse attempts to establish diagnostic protocols and classification systems for spitzoid lesions reflect our scientific "weaknesses" [29,30]. In a recently published study, Geller et al [31] used a Webbased survey to assess clinical use of second opinions and pathologists' perceptions of second opinions for melanocytic lesions. It was not surprising that in daily practice, the majority of pathologists seek second opinions for atypical spitzoid tumors and for melanocytic tumors of uncertain malignant potential. Improvement of interpretive accuracy and protection from medico-legal issues were among the main reasons for requesting a second opinion [31].

In spite of the proposed clinical and histological criteria aimed at distinguishing Spitz nevus from melanoma, in real life a large proportion of these tumors remain a displeasing challenge, for both dermatologist and histopathologist [2833]. In a recent update on dermoscopy of Spitz/Reed nevi by the International Dermoscopy Society, the authors released practical management guidelines for this group of lesions. The consensus-based algorithm recommends that dermoscopically asymmetric lesions (flat, elevated, or nodular) with spitzoid features should be excised to rule out melanoma. Dermoscopically symmetric spitzoid nodules (irrespective of patient age) should be excised, or closely monitored, to rule out atypical Spitz tumors, while flat, dermoscopically 
symmetric, spitzoid lesions should be managed based on patient age. In the end, lesions histopathologically diagnosed as atypical Spitz tumors warrant wide surgical excision, but not a sentinel lymph node biopsy [33].

\section{Conclusions}

We selected 5 clinical examples to highlight different aspects of interest in clinicopathologically problematic melanocytic tumors. In our opinion, in light of the current scientific data, an integrated diagnostic approach that combines the dermatopathological features with the clinical information concerning the evolution of lesions, the patient's profile, and the overall epidemiological characteristics of melanocytic tumors remains the safest course of action.

\section{References}

1. Bombonato C, Ribero S, Pozzobon FC, et al. Association between dermoscopic and reflectance confocal microscopy features of cutaneous melanoma with BRAF mutational status. J Eur Acad Dermatol Venereol. 2017;31(4):643-649.

2. Tschandl P, Berghoff AS, Preusser M, Pammer J, Pehamberger $\mathrm{H}$, Kittler H. Impact of oncogenic BRAF mutations and p16 expression on the growth rate of early melanomas and naevi in vivo. Br J Dermatol. 2016;174(2):364-370.

3. Gonzalez ML, Young ED, Bush J, et al. Histopathologic features of melanoma in difficult-to-diagnose lesions: a case-control study. J Am Acad Dermatol. 2017;77(3):543-548.

4. Ferrara G, Argenziano G, Giorgio CM, Zalaudek I, Kittler H. Dermoscopic-pathologic correlation: apropos of six equivocal cases. Semin Cutan Med Surg. 2009;28(3):157-164.

5. Aung PP, Nagarajan P, Prieto VG. Regression in primary cutaneous melanoma: etiopathogenesis and clinical significance. Lab Invest. 2017 Feb 27;97:657-668.

6. Ribero S, Moscarella E, Ferrara G, Piana S, Argenziano G, Longo C. Regression in cutaneous melanoma: a comprehensive review from diagnosis to prognosis. J Eur Acad Dermatol Venereol. 2016;30(12):2030-2037.

7. Lallas A, Apalla Z, Moscarella E, et al. Extensive regression in pigmented skin lesions: a dangerous confounding feature. Dermatol Pract Concept. 2012;2(2):202a08.

8. Bories N, Dalle S, Debarbieux S, Balme B, Ronger-Savlé S, Thomas L. Dermoscopy of fully regressive cutaneous melanoma. $\mathrm{Br} J$ Dermatol. 2008;158(6):1224-1229.

9. King R, Hayzen BA, Page RN, Googe PB, Zeagler D, Mihm MC. Recurrent nevus phenomenon: a clinicopathologic study of 357 cases and histologic comparison with melanoma with regression. Mod Pathol. 2009;22(5):611-617.

10. Grohs RL, Mesbah Ardakani N. Melanoma manifesting as tumoral melanosis: now you see it, now you don't. Am J Dermatopathol. 2018;40(6):462-465.

11. Izikson L, Sober AJ, Mihm MC Jr, Zembowicz A. Prevalence of melanoma clinically resembling seborrheic keratosis: analysis of 9204 cases. Arch Dermatol. 2002;138(12):1562-1566.

12. Carrera C, Segura S, Aguilera P, et al. Dermoscopic clues for diagnosing melanomas that resemble seborrheic keratosis. JAMA Dermatol. 2017;153(6):544-551.
13. Braun RP, Ludwig S, Marghoob AA. Differential diagnosis of seborrheic keratosis: clinical and dermoscopic features. J Drugs Dermatol. 2017;16(9):835-842.

14. Longo C, Piana S, Marghoob A, et al. Morphological features of naevoid melanoma: results of a multicentre study of the International Dermoscopy Society. Br J Dermatol. 2015;172(4):961-967.

15. Diwan AH, Lazar AJ. Nevoid melanoma. Clin Lab Med. 2011;31(2):243-253.

16. Idriss MH, Rizwan L, Sferuzza A, Wasserman E, Kazlouskaya V, Elston DM. Nevoid melanoma: a study of 43 cases with emphasis on growth pattern. J Am Acad Dermatol. 2015;73(5):836-842.

17. Zembowicz A, McCusker M, Chiarelli C, et al. Morphological analysis of nevoid melanoma: a study of 20 cases with a review of the literature. Am J Dermatopathol. 2001;23(3):167-175.

18. Yélamos O, Busam KJ, Lee C, et al. Morphologic clues and utility of fluorescence in situ hybridization for the diagnosis of nevoid melanoma. J Cutan Pathol. 2015;42(11):796-806.

19. Jaimes N, Marghoob AA, Rabinovitz H, et al. Clinical and dermoscopic characteristics of melanomas on nonfacial chronically sundamaged skin. J Am Acad Dermatol. 2015;72(6):1027-1035.

20. Moscarella E, Argenziano G, Moreno C, et al. Intralesional (incision) biopsy for melanoma diagnosis: the rules and the exception. G Ital Dermatol Venereol. 2017;152(6):658-662.

21. King R, Page RN, Googe PB, Mihm MC Jr. Lentiginous melanoma: a histologic pattern of melanoma to be distinguished from lentiginous nevus. Mod Pathol. 2005;18(10):1397-1401.

22. King R. Lentiginous melanoma. Arch Pathol Lab Med. 2011;135(3):337-341.

23. Wiesner T, Obenauf AC, Murali R, et al. Germline mutations in BAP1 predispose to melanocytic tumors. Nat Genet. 2011;43(10):1018-1021.

24. Ferrara G, Corradin MT. Wiesner nevus. CMAJ. 2017;189(1):E26.

25. Llamas-Velasco M, Pérez-Gónzalez YC, Requena L, Kutzner H. Histopathologic clues for the diagnosis of Wiesner nevus. $J$ Am Acad Dermatol. 2014;70(3):549-554.

26. Yeh I, Mully TW, Wieser T, et al. Ambiguous melanocytic tumors with loss of 3p21. Am J Surg Pathol. 2014;38(8):1088-1095.

27. Njauw C-NJ, Kim I, Piris A, et al. Germline BAP1 inactivation is preferentially associated with metastatic ocular melanoma and cutaneous-ocular melanoma families. PLoS One. 2012;7(4):e35295.

28. Darier J, Civatte A. Naevus ou naevo-carcinoma chez un nourisson. Bull Soc Franc Derm Syph. 1910;21:61-63.

29. Barnhil RL. The spitzoid lesion: rethinking Spitz tumors, atypical variants, 'spitzoid melanoma' and risk assessment. Mod Pathol. 2006;19(suppl 2):S21-S33.

30. Ferrara G, Gianotti R, Cavicchini S, Salviato T, Zalaudek I, Argenziano G. Spitz nevus, Spitz tumor, and spitzoid melanoma: a comprehensive clinicopathologic overview. Dermatol Clin. 2013;31(4):589-598.

31. Geller BM, Frederick PD, Knezevich SR, et al. Pathologists' use of second opinions in interpretation of melanocytic cutaneous lesions: policies, practices, and perceptions. Dermatol Surg. 2018;44(2):177-185.

32. Moscarella E, Lallas A, Kyrgidis A, et al. Clinical and dermoscopic features of atypical Spitz tumors: A multicenter, retrospective, case-control study. J Am Acad Dermatol. 2015;73(5):777-784.

33. Lallas A, Apalla Z, Ioannides D, et al; International Dermoscopy Society. Update on dermoscopy of Spitz/Reed naevi and management guidelines by the International Dermoscopy Society. $\mathrm{Br} J$ Dermatol. 2017;177(3):645-655 